\title{
PROGRAMMABLE CYTOGENETIC SUBMICROLITRE LAB-ON-A-CHIP FOR MOLECULAR DIAGNOSTIC APPLICATIONS
}

\author{
Daniela Woide, Veronika Schlentner, Teresa Neumaier, Thorsten Wachtmeister \\ Herwig G. Paretzke, Zeno von Guttenberg ${ }^{1}$, Achim Wixforth ${ }^{2}$ and Stefan Thalhammer ${ }^{*}$ \\ $G S F$, National Research Center for Environment and Health, Institute of Radiation Protection \\ Ingolstädter Landstrasse 1, 85764 Neuherberg, Germany \\ ${ }^{I}$ Advalytix AG, Sauerbruchstrasse 50, 81377 Munich, Germany \\ ${ }^{2}$ University Augsburg, Chair for Experimental Physics I, Universitätsstrasse 1, 86135 Augsburg, Germany
}

\begin{abstract}
Keywords: Nanobiotechnology, lab-on-a-chip, cytogenetics, microfluidic PCR, surface acoustic waves, laser-based microdissection.

Abstract: This project focuses on the development of an acoustic driven, freely programmable multifunctional biochemical lab-on-a-chip. By combining different platform elements, like microdissection-, nanofluidicand detection-modules, the lab-on-a-chip can be adapted to question- and patient-specific cytogenetic and forensic applications. In contrast to many common lab-on-a-chip approaches presently available, the fluidic handling is done on a planar surface of the lab-on-a-chip. Minute amounts of biochemical fluids are confined in 'virtual' reaction chambers and 'virtual' test tubes in the form of free droplets. The droplets, fluidic tracks and reaction sites are defined at the chip surface by a monolayer chemical modification of the chip surface. Surface acoustic waves are employed to agitate and actuate these little 'virtual' test tubes along predetermined trajectories. Well-defined investigations, controlled in the submicrolitre regime, can be conducted quickly and gently on the lab-on-a-chip.
\end{abstract}

\section{INTRODUCTION}

Over the past decade, advances in molecular biology have helped to enhance understanding of the complex interplay between genetic, transcriptional and translational alterations in, e.g., human cancers. These molecular changes are the basis for an evolving field of high-throughput cancer discovery techniques using microscopic amounts of patientbased material to detect genetic changes such as mutations, insertions, deletions or imbalances.

To be able to reproducibly and reliably handle, process and analyse such small samples, many laboratories all over the world are intensively investigating the applicability of biochips for this purpose. Biochips are small sample carriers, where biological material is attached for analysis. In dependence of the kinds of molecules attached to the surface analytical biochips are divided into DNA-

${ }^{\text {E}}$ The authors wish to be known that, in their opinion the first two authors should be regarded as joint First Author. chips, protein-chips, cell-chips and lab-on-a-chip systems. Most progress in this field occurs especially in the area of DNA-chips (Schneegass et al., 2001; Lagally et al., 2001; Ng and Ilag, 2003). Existing gene chips developed by the Affymetrix Company are a new approach in microarray technology. Oligonucleotide arrays (e.g. genomewide human SNP array as well as human gene array) are based on hybridisation to small, high-density arrays containing tens of thousands of synthetic oligonucleotides. The arrays are designed based on sequence information alone and are synthesized in situ using a combination of photolithography and oligonucleotide chemistry. Chromatin immunoprecipitation (ChiP) coupled with whole-genome DNA-microarrays allows determination of the entire spectrum of in vivo DNA binding sites for any given protein (Buck and Lieb, 2004).

In parallel, another kind of test systems was developed, i.e. bead-technologies (Edelmann et al., 2004) and microfluidic systems (Harrison et al., 
1993; Thorsen et al., 2002; Kwakye and Baeumner, 2003). There is growing interest in performing chemical reactions in microfluidic devices as they offer a variety of significant advantages over macroscopic reactors, such as high surface-area-tovolume ratios and improved control over mass and heat transfer.

Several companies and universities are working on programs employing new electronic DNA analysis technologies. These automated techniques are often developed in non-forensic fields, such as medical research, genetics or biochemistry. In genetic forensics, nucleic acid is usually extracted from saliva, blood, semen, bone, hair and dried skin; these are the sources for most crime scene DNA isolations. Chemical kits for DNA isolation, amplification and detection are available today. The future of forensic testing will follow the path of greater automation e.g. of the DNA fingerprinting process. If a particular kind of polymorphism can be detected through automation, reducing the analysis time and expense, it may be of interest to the forensic community. Developments, however, for forensic applications are rare. Only Nanogen Inc. has developed this technology with applications to STR analysis termed APEX ${ }^{\circledR}$ - automated programmable electronic matrix, by using an electric chip as heart of the analysis (Ibrahim et al., 1998; Rau, 1997).

While DNA-chips become commercially important, scientific and technical development in the last years generated different approaches of multiparameter tests particular for medical applications, so-called 'lab-on-a-chip' systems (LOCs). Miniaturisation of analysis systems will yield in an enormous cost-saving in regard to materials like test tubes or microtitre plates as well as biochemical reagents. Furthermore, a smaller sample volume implies in the end a higher sensitivity and homogeneity of detection. Additionally, in comparison to serial single analyses, parallelisation of analyses enables an enormous time saving due to automation. These micro- and nanolaboratories on the scale of a computer chip are equipped with all components necessary for cytogenetic analysis, they are portable, easy to use, flexible, inexpensive, biocompatible and, like computer chips, full programmable.

Here, we present an acoustic driven lab-on-achip for cytogenetic and forensic applications (Thalhammer et al., 2007). In contrast to many other lab-on-a-chip approaches, the fluidic handling is done on the planar surface of this chip, the fluids being confined in 'virtual' reaction chambers and 'virtual' test tubes in form of free droplets. The droplets, fluidic tracks and reaction sites are defined at the chip surface by a monolayer chemical modification of the chip surface. In comparison to conventional closed microfluidic systems with external pumping, afflicted with the difficulty to further miniaturize, surface acoustic waves are employed to agitate and actuate these little 'virtual' test tubes along predetermined trajectories. These surface acoustic waves propagate on a substrate surface, to move and mix smallest fluidic volumina. Liquid amounts in the range from 1 micro- down to 100 picolitre are precisely moved on monolayers of thin, chemical processed fluidic 'tracks' without any tubing system. The surface acoustic waves are generated by high frequency electrical impulses on microstructured interdigital transducers embedded into the lab-on-a-chip.

Minute amount of sample material is extracted by laser-based microdissection out of e.g. histological sections (Thalhammer et al., 2003; Thalhammer et al., 2004). A few picogram of genetic material are isolated and transferred via a low-pressure transfer system onto the lab-on-a-chip. Subsequently the genetic material inside single droplets, which behave like 'virtual' beakers, is transported to the reaction and analysis centres on the chip surface via surface acoustic waves, probably best known from their use as high frequency filters in mobile phones. At these 'biological reactors' the genetic material is processed, e.g. amplified via polymerase chain reaction methods, and genetically characterized (Guttenberg et al., 2005).

Well-defined analyses, controlled in the submicrolitre regime, can be quickly and gently conducted on the lab-on-a-chip. Apart from its nearly unlimited applicability for many different biological assays, its programmability and extremely low manufacturing costs are another definite advantage of this 'cytochip'. In fact, those LOCs can be made so cheap that their use as disposables in many areas of diagnostics can be envisioned.

\section{MATERIAL \& METHODS}

In most microfluidic systems liquids are confined and moved in tubes or capillaries. Usually, the application of such systems is restricted to continuous flow processes. However, when carefully looking at a microscale fluid, one realizes that the effects of e.g. surface tension by far exceed those of gravity. The shape of a droplet on a surface is given 
by the properties of the substrate. It either remains a droplet or it wets the surface, depending on whether the substrate is hydrophobic or hydrophilic. The technology to create such fluidic tracks (fig. 1) is very much similar to define conducting paths on an electronic semiconductor device.

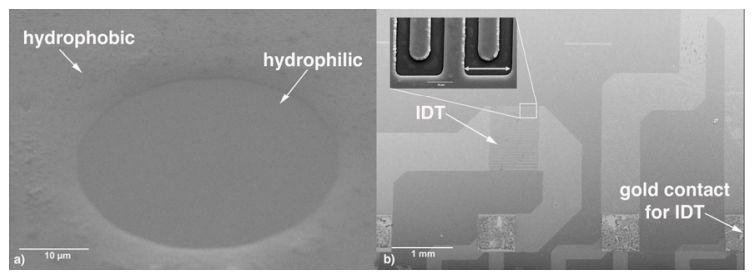

Figure 1: Chemical functionalization of the chip surface creating a hydro-philic/hydrophobic structure. Electron microscopy of a) the hydrophilic reaction centre on the hydrophobic LOC surface, scale bar $10 \mu \mathrm{m}$, and b) the arrangement of interdigital transducers (IDT) and electric conduction to control the SAW, scale bar $1 \mathrm{~mm}$. Enlargement in b), electron microscopy of the interdigital transducer in detail with comb periodicity of the doubleheaded arrow, scale bar $10 \mu \mathrm{m}$.

Small amounts of liquids do not really need to be confined in tubes and trenches. They form their own test tubes, held together by surface tension effects. These micro volumina, due to the fact that in small droplets the effect of surface tension dominates gravity, do not need any reservoir as surface tension keeps the droplets in shape. Visualizing dewdrops hanging on a spider's web, one can observe that average droplet size obviously depends on the thickness of the strand: smaller droplets are attached to finer fibres and bigger ones to thicker threads. Apparently droplet shape conforms to the geometry as well as the wettability of the subsurface.

A 'lab-on-a-chip', however, requires more than just test tubes. More important, their cargo has to be moved around, mixed, stirred or processed in general.

\subsection{Lab-on-a-Chip Design}

The layout of the lab-on-a-chip is shown layer by layer in a schematic drawing (fig. 2). The basic material of the lab-on-a-chip is a lithium niobate $\left(\mathrm{LiNbO}_{3}\right)$ single crystal wafer polished on both sides. The first metal layer is platinum (Pt) or nickel (Ni) for the heater and sensor structure, followed by a gold layer for the SAW transducer and the contact wires. The complete chip is protected with sputter oxide, which is removed above the contact pads. All structures are patterned by photolithography.

A chemical functionalization of the surface or parts thereof can be employed to laterally define a modulation of the wetting properties, thus creating fluidic pathways or tracks forming virtual potential wells for a fluid on the flat surface of a chip (fig. 3). To form a high contact angle of the oil on the chips, the surface has to be lipophilic. However, the hybridization array has to be wetted easily and needs active coupling groups for the oligo DNA spots. Therefore a chemically heterogeneous surface modification is needed achieved by photolithography. The tracking system for biochemical reactants and oil droplet movement and heaters on the chip is patterned with photoresist. An organic layer of a hydrophobic silane is bound to the whole surface. After removing the photoresist, epoxysilane is grafted from an organic solution.

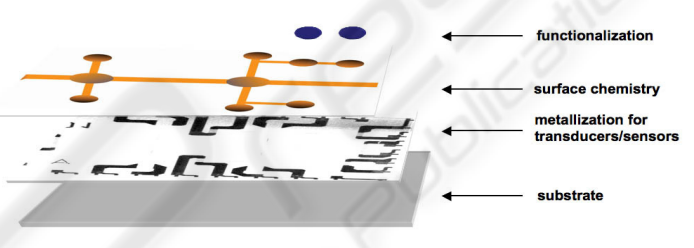

Figure 2: Design of LOC functionality. The ground substrate $\left(\mathrm{LiNbO}_{3}\right)$ is covered by a layer of $\mathrm{Pt}, \mathrm{Ni}$ and $\mathrm{Au}$ for transducers and sensor metallization. Subsequent silanisation of the surface accounts for a hydrophilic/hydrophobic surface chemistry, facilitating a planar tracking system, which could be further functionalised.

\subsection{Actuation}

Actuation of single droplets or closed loops of liquid on a fluidic track is achieved by so-called surface acoustic waves, which have been widely used in the completely different field of radio frequency signal processing over the last twenty years or so. Each cell phone, for instance, contains two or more devices operating on SAW.

Actuation of small droplets on the surface of a SAW chip is caused by the effect of acoustic streaming. This phenomenon appears when intensive sound fields are travelling through a liquid. Two major actuation forms can be described: internal flow inside the droplet versus transport of the droplet.

\subsubsection{Interdigital Transducer}

Electronic devices employing the SAW normally utilize one or more interdigital transducers (IDTs) to convert acoustic waves to electrical signal and vice versa utilizing the piezo-electric effect of certain materials (i.e. $\mathrm{LiNbO}_{3}$ ) (fig. 1). These devices are 
fabricated utilizing common processes used in the manufacture of silicon integrated circuits. Piezoelectricity is the ability of crystals to generate a voltage in response to applied mechanical stress. Depending on their design, the interdigital transducers produce a special type of acoustic surface wave, which can efficiently transfer energy into liquids. Typical SAW frequencies for the fluidic application presented here range from 100 to $200 \mathrm{MHz}$, the wavelengths are then around 20 micrometers. Transducers are copied from a transducer mask (Advalytix); a distance of $26.5 \mu \mathrm{m}$ between two combs results in a resonance frequency on the $\mathrm{LiNbO}_{3}$ of $150.6 \mathrm{MHz}$ (fig. 1).
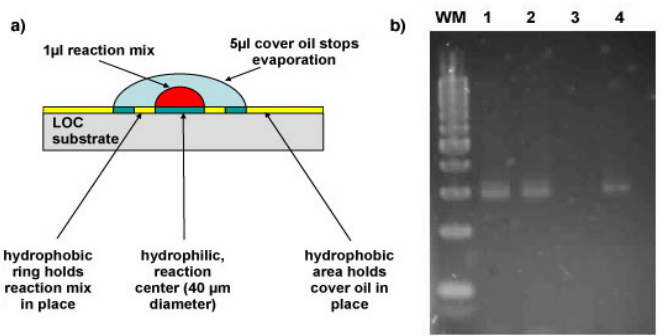

Figure 3: a) Side-view of the lab-on-a-chip amplification unit displaying single droplet PCR. The PCR reaction mix $(1 \mu 1)$ on the hydrophilic reaction centre $(40 \mu \mathrm{m}$ in diameter) is covered with mineral oil to avoid evaporation. The hydrophobic area around the reaction centre holds reaction mix and cover oil in place. b) Gel electrophoresis of $1 \mu 1 \beta$-Actin PCR. WM: weight marker, PeqGold 100 bp DNA ladder; lane 1+2: positive control; lane 3: negative probe; lane 4: LOC-PCR, 500 pg target DNA.

\subsubsection{Surface Acoustic Wave}

Surface waves, so-called Rayleigh-waves, are applied on the piezo-electric system without any mechanical contact to realize actuation of the reactants on the LOC with interdigital transducers.

A surface acoustic wave is an acoustic wave travelling along the surface of a material having some elasticity, with amplitude that typically decays exponentially with the depth of the substrate. It is the nanometre analogue of an earthquake. This kind of wave is commonly used in piezo-electric devices called SAW devices in electronic circuits. Its amplitude and wavelength, however, can be controlled by an electrical signal applied to an appropriate transducer.

At low amplitudes, e.g. below one nanometre, a striking SAW pulse creates internal streaming within the fluid. Its energy is strongly absorbed and radiated into the fluid under the Rayleigh angle. At larger amplitudes, the internal streaming becomes a movement of the whole droplet into the desired direction on the chip with a desired speed (Wixforth et al., 2004). Velocities close to one $\mathrm{m} / \mathrm{sec}$ can be achieved in this way. In this sense, the transducer generating the surface acoustic waves can be regarded as pump without moving parts that may be remotely operated to control the position of one or more single droplets on the planar fluidic network on a LOC system.

\section{PROTOTYPE}

Here, we present a multifunctional lab-on-a-chip combining different platform elements like microdissection-, nanofluidic- and detectionmodules (fig. 4) with the aim of providing a new platform for fast, cheap and easy investigation of genetic material in patient or forensic samples.

Without any mechanical structuring the lab-on-achip exhibits 'virtual' tracks, whereon samples and reagents are acoustically driven, actuated by electrical nanopumps (fig. 5). For the LOC, specific reaction-predefined 'spots' can be generated for total genome amplification via PCR, labelling of the amplified material and detection.

The recent developed lab-on-a-chip system combines serial processing with parallel downstream applications by using a minimum amount of genetic material as source for further investigation. Amplification, labelling and detection of the isolated genetic material are subsequently carried out on the chip surface driven by surface acoustic waves.

Each lab-on-a-chip (fig. 5) has two areas operating as biochemical reaction points, controlled by the temperature sensor. The sensors of the chip are calibrated by a thermoplate and resistance measurements controlled by a LabView program. The chip has 10 separately addressable SAW transducers, two on each side for aligning the reactant droplet on the heaters and for mixing during the biochemical reactions. Opposing transducers have different spatial periods to avoid crosstalk.

Extraction of sample material is performed via laser-based microdissection providing the possibility to isolate samples in the range from several cells down to a single chromosomal band with minimum risk of contamination. These small amounts of genetic material, which lay in the range of several picogram, are then transferred via a low-pressure transfer system onto the lab-on-a-chip. This newly developed transfer system (publication in preparation) extracts microdissected material using 


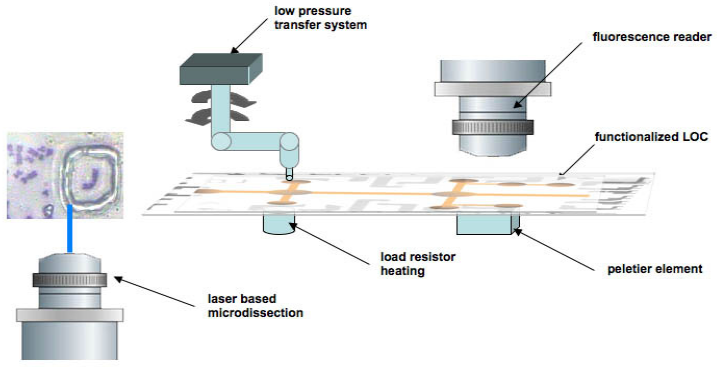

Figure 4: The modular lab-on-a-chip system consists of several units for isolating, processing and analysing minute amounts of sample material: laser-based microdissection is followed by processing of the extracted material and detection of hybridized probes or amplified material by a fluorescence reader. All operations on the LOC are controlled by SAW actuated microfluidics.

low-pressure and transfers it to the reaction centre on the LOC. The patented transfer device allows the precise positioning of the isolated material on top of the LOC.

Subsequently, the sample material inside single droplets is transported very efficiently and contactless to the reaction and analysis centres on the chip surface via surface acoustic waves. Processing of isolated genetic material like specific or unspecific amplification is conducted on the nanofluidic device via polymerase chain reaction methods followed by labelling with fluorochromes.

Qualitative as well as quantitative analyses such as real-time PCR or microarray will be carried out using a novel 'fluorescence reader', especially designed for the LOC system and forming the principal component of the detection unit. Thus different applications for point-of-care diagnostics are practicable on one single lab-on-a-chip.

\section{POSSIBLE APPLICATIONS}

This freely programmable lab-on-a-chip system will open new potentials in research and development in different fields of applications ranging from cytogenetics to pathology and forensics.

Apart from its nearly unlimited applicability for many different biological assays, possible applications of this system in cytogenetics are e.g. detection of chromosomal imbalances and detection of genomic imbalances in solid tumour tissue. After isolation of the genetic material by laser-based microdissection the sample is transferred to the labon-a-chip using a low- pressure transfer system. Furthermore, in a first biochemical reaction the extracted material is enzymatically digested and prepared for subsequent amplification e.g. Alu PCR for SNP analyses. Whole genome amplification of e.g. individually isolated single cells and chromosomes followed by labelling the material with fluorochromes can be used for e.g. fluorescence in situ hybridization (FISH) experiments. After mixing with different-labelled reference-DNA the mixture can be transferred to the specific CGH array via wetting modulated surface chemistry, hybridized and finally detected. To make sure that fluorochromes are incorporated uniformly into the sample DNA as well as the reference DNA, the labelling process can be monitored using online PCR detection. In the same way the amount of DNA product after amplification can be determined exactly. This provides the possibility to mix equal amounts of sample and reference DNA for the hybridization mixture. The detection array, microarray on the lab-on-a-chip, will be questionand patient-specific spotted by dot blot technology. Again acoustic actuation will be adopted to solve e.g. lyophilized reagents in a specific buffer.

With regard to the emerging field of forensics, this LOC system can also be applied to genomic sample material as blood cells, buccal swaps or other human cell material performing DNA fingerprint, paternity tests or SNP analysis. As forensic analysis should be cost and time saving, the development of a new miniature analytical lab-on-a-chip system could serve the market in a novel and promising way. Real-time PCR and STR analysis could not only be applied to the novel LOC system separately but also be combined on one single lab-on-a-chip in a modular way.

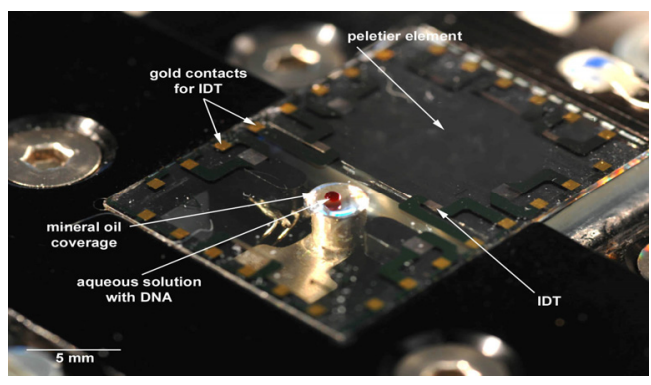

Figure 5: SAW driven lab-on-a-chip system with 10 interdigital transducers, two heaters and biochemical reactant containers. Transport of minute amounts of sample material in 'virtual' beakers is actuated by surface acoustic waves generated via interdigital transducers. The liquid phase comprising the genetic material (red) is covered by a thin layer of mineral oil avoiding evaporation. A load resistor heating and a peltier element provide for precise temperature profiles required for molecular biological methods. 
A further application in the field of systems radiation biology is to investigate the influence of low-dose irradiation on cell-cell interactions and possible bystander effects. The medical use of ionizing radiation contributes the largest fraction to the population's anthropogenic radiation exposure. Thus, biopsies of suspicious diagnostic findings, which were irradiated with standard low-dose, will be extracted for histological examination and isolated cell clusters will be analysed on the LOC. By spotting a specific protein array on one part of the LOC and moving cells and media via SAW onto this particular array, it should be possible to detect cancer cascades and involved proteins. This method can be further used to determine the relation and interaction between cancer associated proteins i.e. p53, TGF- $\beta$ and caspase.

\section{OUTLOOK}

This system, the acoustically driven, freely programmable multifunctional biochemical lab-ona-chip system, will be applied on different diagnostic approaches at the single cell or single chromosome level e.g. cytogenetics, tumour genetics and genetic forensics.

Competitive LOCs, combining these techniques, are worldwide not on the market. An essential advantage of the LOC system is the modular set-up, which allows reacting to different diagnostic questions in a preventive medical check-up. This implements the rapid adaptation to patient-specific point-of-care diagnostics as well as the operatorspecific development of new molecular markers for imaging techniques. Fields of applications for the newly developed LOC system range from the analysis of cell compartments and single cells in tumour diagnosis to chromosomal imbalances in the human genome.

\section{ACKNOWLEDGEMENTS}

The authors would like to thank Klaus Macknapp, Deutsches Museum Munich, for providing the electron microscopy images.

Financial support of the Bavarian Research Foundation, Deutsche Forschungsgemeinschaft (DFG) SFB 486 grant and German Excellence Initiative via the 'Nanosystems Initiative Munich (NIM)' is gratefully acknowledged.

\section{REFERENCES}

Buck M.J. and Lieb J.D., 2004. ChiP-chip: considerations for the design, analysis, and application of genomewide chromatin immunoprecipitation experiments. Genomics 83: 349-360

Edelmann, L., Hashmi, G., Song, Y., Han, Y., Kornreich, R., Desnick, R.J., 2004. Cystic fibrosis screening: validation of a novel method using Bead Chip technology. Genet Med. 6(5): 431-438

Guttenberg, Z., Müller, H., Habermüller, H., Geisbauer, A., Pipper, J., Felbel, J., Kielpinski, M., Scriba, J., Wixforth, A., 2005. Planar chip device for PCR and hybridization with surface acoustic wave pump. Lab on a chip 5: 308-317

Harrison, D.J., Fluri, K., Seiler, K., Fan, Z., Effenhauser, C.S., Manz, A., 1993. Micromaching a miniaturized capillary electrophoresis-based chemical analysis system on a chip. Science 261: 895-897

Ibrahim, M.S., et al., 1998. Real-time microchip PCR for detecting single-base differences in viral and human DNA. Anal Chem. 70: 2013

Kwakye, S., Baeumner, A., 2003. A microfluidic biosensor based on nucleic acid sequence recognition. Anal. Bioanal. Chem. 376(7): 1062-1068

Lagally, E.T., Emrich, C.A., Mathies, R.A., 2001. Fully integrated PCR-capillary electrophoresis microsystem for DNA analysis. Lab on a chip 1: 102-107

Ng, J.H., Ilag, L.L., 2003. Biochips beyond DNA: technologies and applications. Biotechnol. Annu. Rev. 9: 1-149

Rau, R., 1997. The National Institute of Justice Forensic DNA Laboratory Improvement Program. Profiles DNA 1: 5

Schneegass, I., Brautigam, R., Kohler, J.M., 2001. Miniaturized flow-through PCR with different template types in a silicon chip thermocycler. Lab Chip 1(1): $42-49$

Thalhammer, S., Lahr, G., Clement-Sengewald, A., Heckl, W.M., Burgemeister, R., Schütze, K., 2003. Laser microtools in cell biology and molecular medicine. Laser Physics 13(5): 681-692

Thalhammer, S., Langer, S., Speicher, M.R., Heckl, W.M., Geigl, J.B., 2004. Generation of chromosome painting probes from single chromosomes by laser microdissection and linker-adaptor PCR. Chromosome Research 12: 337-343

Thalhammer, S., von Guttenberg, Z., Koehler, U., Zink, A., Heckl, W.M., Franke, T., Paretzke, H.G., Wixforth, A., 2007. Programmierbares, zytogenetisches Submikroliter-Chiplabor für molekular-diagnostische Anwendungen. GenomXPress ISBN: 1617-562X, 1.07: 29-31

Thorsen, T., Maerkl, S.J., Quake, S.R., 2002. Microfluidic large-scale integration. Science 298: 580-584

Wixforth, A., Strobl., C., Gauer, C., Toegl, A., Scriba, J., Guttenberg, Z., 2004. Acoustic manipulation of small droplets. Anal. Bioanal. Chem. 379: 982-991 